\title{
Nonsymmetrized hyperspherical harmonics with realistic NN potentials
}

\author{
Sergio Deflorian ${ }^{1,2}$, Nir Barnea ${ }^{3}$, Winfried Leidemann ${ }^{1,2}$, and Giuseppina Orlandini ${ }^{1,2}$ \\ ${ }^{1}$ Dipartimento di Fisica, Università di Trento, I-38123 Trento, Italy \\ ${ }^{2}$ Istituto Nazionale di Fisica Nucleare, \\ Gruppo Collegato di Trento, I-38123 Trento, Italy \\ ${ }^{3}$ Racah Institute of Physics, The Hebrew University, 91904, Jerusalem, Israel
}

\begin{abstract}
The Schrödinger equation is solved for an $A$-nucleon system using an expansion of the wave function in nonsymmetrized hyperspherical harmonics. Our approach is both an extension and a modification of the formalism developed by Gattobigio et al. [1, 2]. The extension consists in the inclusion of spin and isospin degrees of freedom such that a calculation with more realistic NN potential models becomes possible, whereas the modification allows a much simpler determination of the fermionic ground state. The approach is applied to four- and six-body nuclei $\left({ }^{4} \mathrm{He},{ }^{6} \mathrm{Li}\right)$ with various NN potential models. It is shown that the results for ground-state energy and radius agree well with those from the literature.
\end{abstract}




\section{INTRODUCTION}

Expansions of the wave function of an $A$-nucleon system on a specific basis set is a common tool in few-nucleon ab initio calculations (for an overview see Ref. [3]). One of the challenges in such calculations is the implementation of the proper permutation symmetry, i.e. of the antisymmetry for a fermion system like the atomic nucleus. A frequently used basis are the hyperspherical harmonics $(\mathrm{HH})$. For a growing particle number an effective HH symmetrization method had been developed in Ref. [4]. The method, however, requires considerable computational resources with respect to memory and cpu time. Recently a different $\mathrm{HH}$ approach has been proposed in order to construct wave functions with a proper permutation symmetry [1, 2]. It consists in the use of a nonsymmetrized HH (NSHH) basis thus avoiding the HH symmetrization procedure. Since the Hamiltonian commutes with the permutation operator, all non degenerate eigenstates of the Hamiltonian have a well defined permutation symmetry. The disadvantage is that additional effort has to be devoted to the determination of the respective symmetry. On the other hand, in view of an application of the $\mathrm{HH}$ expansion method beyond $A=4$, the use of NSHH might be advantageous.

In this work we extend the NSHH to consider spin and isospin degrees of freedom, which allows us to work with modern realistic NN potentials. In addition we show how the antisymmetric ground state can be identified in a very simple and effective way. We apply our technique to calculate ground-state energies and radii of ${ }^{4} \mathrm{He}$ and ${ }^{6} \mathrm{Li}$ with various NN potential models. In case of ${ }^{4} \mathrm{He}$ we also use the modern realistic AV18 potential [5].

Our paper is organized as follows. The HH basis with inclusion of spin and isospin degrees of freedom is discussed in Section [II. The formalism of Ref. [1, 2] for an efficient use of a nonsymmetrized $\mathrm{HH}$ basis is laid out in the first part of Section III, whereas our modifications of the method are outlined in the second part of Section III Our results for ${ }^{4} \mathrm{He}$ and ${ }^{6} \mathrm{Li}$ are discussed in Section [IV, which also includes a brief summary. 


\section{THE HH BASIS}

To describe the HH basis, we first introduce the Jacobi coordinates. Here we use them in reversed order and for particles with equal mass,

$$
\boldsymbol{\eta}_{i}=\sqrt{\frac{A-i}{A+1-i}}\left(\boldsymbol{r}_{i}-\frac{1}{A-i} \sum_{j=i+1}^{A} \boldsymbol{r}_{j}\right), \quad i=1, \ldots, N
$$

where $A$ is the number of particles, $N=A-1$, and $\boldsymbol{r}_{i}$ is the position vector for the $i$-th

particle. To each Jacobi vector $\boldsymbol{\eta}_{i}$ an angular momentum operator $\hat{\boldsymbol{l}}_{i}$ is associated. For the total orbital angular momentum operator up to the $n$-th coordinate $(n=2, \ldots, N)$ one has

$$
\hat{\boldsymbol{L}}_{n}=\hat{\boldsymbol{L}}_{n-1}+\hat{\boldsymbol{l}}_{n}
$$

with $\hat{\boldsymbol{L}}_{1} \equiv \hat{\boldsymbol{l}}_{1}$. The hyperradial and the hyperangular variables $\rho_{n}$ and $\theta_{n}$ are defined as

$$
\sin \theta_{n}=\frac{\eta_{n}}{\rho_{n}}, \quad \rho_{n}=\sqrt{\sum_{i=1}^{n} \eta_{i}^{2}}, \quad n=2, \ldots, N .
$$

The $3 N$ variables $\left\{\boldsymbol{\eta}_{1}, \ldots, \boldsymbol{\eta}_{N}\right\}$ are thus replaced by $\left\{\rho, \Omega_{N}\right\}$, i.e. one hyperradial coordinate $\rho \equiv \rho_{N}$ and $3 N-1$ hyperangular coordinates $\Omega_{N}=\left\{\hat{\eta}_{1}, \ldots, \hat{\eta}_{N}, \theta_{2}, \ldots, \theta_{N}\right\}$. The following relation,

$$
\rho=\sqrt{\frac{1}{A} \sum_{j>i=1}^{A}\left(\boldsymbol{r}_{j}-\boldsymbol{r}_{i}\right)^{2}}
$$

shows that the hyperradius is symmetric under permutation of particles.

Using the above set of coordinates, the Laplace operator for $n=1, \ldots, N$ can be rewritten as

$$
\begin{aligned}
\Delta_{n}=\sum_{i=1}^{n} \nabla_{\eta_{i}}^{2} & =\frac{1}{\rho^{3 n-1}} \frac{\partial}{\partial \rho} \rho^{3 n-1} \frac{\partial}{\partial \rho}-\frac{1}{\rho^{2}} \hat{K}_{n}^{2} \\
& =\frac{\partial^{2}}{\partial \rho^{2}}+\frac{3 n-1}{\rho} \frac{\partial}{\partial \rho}-\frac{1}{\rho^{2}} \hat{K}_{n}^{2}
\end{aligned}
$$

where $\hat{K}_{n}^{2}\left(\Omega_{n}\right)$ is a generalization of the usual angular momentum operator $\hat{K}_{1}^{2} \equiv \hat{l}_{1}^{2}$ and is called the grand angular momentum operator. Its explicit expression for $n \geq 2$ is given by

$$
\begin{aligned}
\hat{K}_{n}^{2}= & -\frac{\partial^{2}}{\partial \theta_{n}^{2}}+\frac{3 n-6-(3 n-2) \cos \left(2 \theta_{n}\right)}{\sin \left(2 \theta_{n}\right)} \frac{\partial}{\partial \theta_{n}} \\
& +\frac{1}{\cos ^{2} \theta_{n}} \hat{K}_{n-1}^{2}+\frac{1}{\sin ^{2} \theta_{n}} \hat{l}_{n}^{2} .
\end{aligned}
$$


The hyperspherical harmonic functions $\mathcal{Y}_{[K]}\left(\Omega_{N}\right)$ are the eigenfunctions of the grand angular momentum operator:

$$
\left[\hat{K}_{N}^{2}\left(\Omega_{N}\right)-K(K+3 N-2)\right] \mathcal{Y}_{[K]}\left(\Omega_{N}\right)=0
$$

The $\mathrm{HH}$ can be expressed in terms of the spherical harmonics $Y_{l m}(\hat{\eta})$ and of the Jacobi polynomials $P_{\mu}^{a, b}(z)$ :

$$
\begin{aligned}
\mathcal{Y}_{[K]}\left(\Omega_{N}\right)= & {\left[\sum_{m_{1}, \ldots, m_{N}}\left\langle l_{1} m_{1} l_{2} m_{2} \mid L_{2} M_{2}\right\rangle\left\langle L_{2} M_{2} l_{3} m_{3} \mid L_{3} M_{3}\right\rangle \ldots\right.} \\
& \left.\times\left\langle L_{N-1} M_{N-1} l_{N} m_{N} \mid L_{N} M_{N}\right\rangle \prod_{j=1}^{N} Y_{l_{j} m_{j}}\left(\hat{\eta}_{j}\right)\right] \\
& \times\left[\prod_{j=2}^{N} \mathcal{N}\left(K_{j} ; l_{j} K_{j-1}\right)\left(\sin \theta_{j}\right)^{l_{j}}\left(\cos \theta_{j}\right)^{K_{j-1}}\right. \\
& \left.\times P_{\mu_{j}}^{\left[l_{j}+1 / 2\right],\left[K_{j-1}+(3 j-5) / 2\right]}\left(\cos \left(2 \theta_{j}\right)\right)\right] .
\end{aligned}
$$

The coefficients $\mathcal{N}_{j}\left(K_{j} ; L_{j} K_{j-1}\right)$ are normalization coefficients given by

$$
\mathcal{N}_{j}\left(K_{j} ; L_{j} K_{j-1}\right)=\left[\frac{\mu_{j} !\left(2 K_{j}+3 j-2\right) \Gamma\left(\mu_{j}+K_{j-1}+l_{j}+\frac{(3 j-2)}{2}\right)}{\Gamma\left(\mu_{j}+l_{j}+\frac{3}{2}\right) \Gamma\left(\mu_{j}+K_{j-1}+\frac{(3 j-2)}{2}\right)}\right]^{1 / 2},
$$

where the numbers $\mu_{j}$ are non-negative integers, and $K_{j}=K_{j-1}+2 \mu_{j}+l_{j}$.

The basis functions consist not only of the hyperangular part, but also of the hyperradial part and of the spin and isospin part,

$$
\left|\Phi_{i}\right\rangle=\left|\mathcal{R}_{r_{i}} \mathcal{Y}_{[K]_{i}}\right\rangle \otimes\left|\chi_{[S]_{i}}^{\mathrm{spin}} \chi_{[T]_{i}}^{\text {isospin }}\right\rangle
$$

where $i$ enumerates the basis state $\Phi_{i}$, and $\mathcal{R}_{r}$ are the hyperradial functions numbered by the index $r$ (we use Laguerre polynomials). The hyperspherical harmonic functions $\mathcal{Y}_{[K]}$ are identified by the following set of quantum numbers

$$
[K]=\left\{K_{N}, K_{N-1}, \ldots, K_{2}, L_{N}, L_{N-1}, \ldots, L_{2}, l_{N}, l_{N-1}, \ldots, l_{2}, l_{1}\right\}
$$

and $\chi_{[S]}^{\text {spin }}, \chi_{[T]}^{\text {isospin }}$ are the spin and isospin basis states, respectively, defined by the sets of quantum numbers

$$
\begin{aligned}
& {[S]=\left\{S_{A}, S_{A-1}, \ldots, S_{2}\right\}} \\
& {[T]=\left\{T_{A}, T_{A-1}, \ldots, T_{2} ; T_{A, z}\right\} .}
\end{aligned}
$$


The spin quantum number $S_{j}$ is the value of the coupled spin momenta of particles 1 to $j$. The isospin states are characterized in a similar way with the addition of $T_{A, z}$, the third component of the total isospin $T_{A}$.

In order to consider potentials that depend on spin and isospin, one has to take as basis states the complete functions $\left|\Phi_{i}\right\rangle$, and so one has to consider the whole set of quantum numbers $\{[K],[S],[T]\}$ limited by a maximal value $K_{\max }$ of $K_{N}$. Two possibilities arise: if the potential is central, then not only the total angular momentum $J$, the total isospin $T=T_{A}$ (isospin mixing neglected) and its projection $T_{A, z}$ are good quantum numbers for the eigenstates of $H$, but also the total orbital angular momentum $L=L_{N}$ and therefore the total spin $S=S_{A}$ (in fact $J$ becomes obsolete). In this case the total number of basis states is given by the number of radial functions times the number of hyperspherical harmonic functions times the number of spin-isospin functions, subject to the condition that $S, L, T$ and $T_{A, z}$ have the desired values. On the other hand, if the potential is noncentral, like for example the realistic potential AV18, $L$ and $S$ are no longer good quantum numbers, leaving only $J, T$ and $T_{A, z}$ as constraints in the construction of the basis, and of course leading to a higher number of basis states. The size of the basis is the limiting factor in the applicability of the method. Examples for the number of basis states are presented in Section IV.

\section{EXPANSIONS WITH NONSYMMETRIZED HH FUNCTIONS}

In order to describe the nonsymmetrized $\mathrm{HH}$ (NSHH) method introduced by Gattobigio et al. [1, 2], we first discuss a few aspects concerning permutation symmetry. The HH functions defined above do not have well-defined permutational symmetries. A particle permutation changes the definition of the Jacobi coordinates as well as the couplings between the different angular momenta. Consequently, the effect of particle permutation on the $\mathrm{HH}$ functions is rather complicated, and the matrices representing the permutation operators $\hat{P}_{i j}$ on the $\mathrm{HH}$ basis are arbitrary matrices (not diagonal or block-diagonal matrices), and have to be calculated numerically. In order to reduce the evaluation of the matrix elements of the potential acting between particles $i$ and $j$ into a one dimensional integral one has to use Jacobi vectors such that $\boldsymbol{\eta}_{A-1}=\sqrt{\frac{1}{2}}\left(\boldsymbol{r}_{i}-\boldsymbol{r}_{j}\right)$. This transformation can be realized through the permutations $\hat{P}_{i, A-1}$ and $\hat{P}_{j, A}$ acting on the $\mathrm{HH}$ basis states. The matrix representing 
the permutation operator $\hat{P}_{i j}$ can be written as

$$
\mathcal{B}_{[K, S, T]\left[K^{\prime}, S^{\prime}, T^{\prime}\right]}^{i j}=\left\langle\Phi_{[K, S, T]}\left(\Omega_{N}^{i j}\right) \mid \Phi_{\left[K^{\prime}, S^{\prime}, T^{\prime}\right]}\left(\Omega_{N}\right)\right\rangle,
$$

where the set of quantum numbers $[K, S, T] \equiv\{[K],[S],[T]\}$ identify the orbital, spin and isospin quantum numbers of the basis functions, $\Omega_{N}$ is the set of all hyperangular variables defined above and $\Omega_{N}^{i j}$ indicates the set of hyperangular variables with the particles $i$ and $j$ interchanged. All permutations can be expressed as a product of a certain number $\nu_{i j}$ of transpositions (depending on the particles indexes $i$ and $j$ ) in which only adjacent particles are interchanged,

$$
\hat{P}_{i j}=\prod_{l=1}^{l=\nu_{i j}} \hat{P}_{k_{l}}=\hat{P}_{k_{1}} \ldots \hat{P}_{k_{\nu_{i j}}}
$$

where the operator $\hat{P}_{k}$ exchanges particles $k$ and $k+1$. This can be interpreted as follows: each particle carries a number and occupies originally a a box with the same number. To bring particle number $i$ in the box $j$ and vice versa, one can act with a certain number $\nu_{i j}$ of permutations that exchange only particles in adjacent boxes. Thanks to the properties of the HHs, the matrices

$$
\mathcal{B}_{[K, S, T]\left[K^{\prime}, S^{\prime}, T^{\prime}\right]}^{k, k+1}=\left\langle\Phi_{[K, S, T]}\left(\Omega^{k, k+1}\right) \mid \Phi_{\left[K^{\prime}, S^{\prime}, T^{\prime}\right]}\left(\Omega_{N}\right)\right\rangle
$$

representing the operators $\hat{P}_{k}$ are block diagonal and can be easily calculated. This provides an easy and fast way to calculate the product of the $\mathcal{B}$ matrices on state vectors.

We remark that the use of the NSHH has been developed in order to avoid the need for the symmetrization of the basis functions, which, as already pointed out in the introduction, requires non negligible computational resources. Though the number of basis functions for equal values of $K_{\max }$ is in general considerably larger in the NSHH method than with symmetrized functions, it might be still advantageous to use nonsymmetrized functions.

In Ref. [2] bound states have been calculated using the Volkov potential [6], which is central and independent on spin and isospin. In this case the spatial wave function can be treated separately from the spin-isospin part. The Hamiltonian is represented on the $\mathrm{HH}$ and diagonalized. Then the symmetry of the eigenstates is analyzed and the spin-isospin part with the correct symmetry is multiplied in order to obtain an antisymmetric wave function. It is clear that following this procedure the number of $\mathrm{HH}$ basis states, and therefore the size of the Hamiltonian matrix to be diagonalized, is relatively low. However, this procedure only applies to potentials that do not depend on spin and isospin. 
In the following we describe how to modify the method outlined above in order to be able to treat also realistic potentials. In this case we need to deal with basis functions in the Jcoupling as described in Section II. This leads to an increase in the size of the Hamiltonian matrix and therefore in the number of eigenfunctions that need to be analyzed in terms of symmetry. In order to speed up the search for the antisymmetric ground state we have implemented a method based on the use of the transposition class sum operator, the Casimir operator, of the permutation group. This method is analogous to the Lawson method [7] for the removal of the spurious center of mass motion in Shell Model calculations.

The Casimir operator of the permutation group $\hat{C}(A)=\sum_{j>i=1}^{A} \hat{P}_{i j}$ and the Hamiltonian $H$ commute; hence they can be diagonalized simultaneously. In general, the eigenvalues of the operator $\hat{C}(A)$ are not sufficient to identify the irreducible representations of the permutation group. However, the completely symmetric and antisymmetric representations correspond to the extreme eigenvalues of $\hat{C}(A)$ and are well separated from the rest of the spectrum. Antisymmetric states correspond to the lowest eigenvalue of $\hat{C}(A)$, while symmetric states correspond to the highest one, in detail one has

$$
\begin{aligned}
& \hat{C}(A) \Psi_{S}=\frac{A(A-1)}{2} \Psi_{S}=\lambda_{S} \Psi_{S}, \\
& \hat{C}(A) \Psi_{M}=\lambda_{M} \Psi_{M}, \\
& \hat{C}(A) \Psi_{A}=-\frac{A(A-1)}{2} \Psi_{A}=\lambda_{A} \Psi_{A},
\end{aligned}
$$

where $\Psi_{S}, \Psi_{M}, \Psi_{A}$, correspond to symmetric, mixed-symmetry, and antisymmetric states, respectively, and $\lambda_{A}<\lambda_{M}<\lambda_{S}$. We diagonalize the matrix

$$
H^{\prime}=H+\gamma \hat{C}(A)
$$

where $\gamma$ is a real parameter. The eigenvalues of $H^{\prime}$ are given by

$$
E_{n, \Gamma}^{\prime}=E_{n, \Gamma}+\gamma \lambda_{\Gamma}
$$

where $E_{n, \Gamma}\left(n=0,1,2, \ldots, N_{\max }(\Gamma)\right)$ are the eigenvalues of $H$ for the symmetry $\Gamma$ $(\Gamma=S, M, A)$. We denote by $\mathcal{E}$ the lowest eigenvalue of the Hamiltonian $H(\mathcal{E}=$ $\left.\min \left\{E_{0, S}, E_{0, M}, E_{0, A}\right\}\right)$, which can be found by performing the calculation with $\gamma=0$.

To calculate the lowest antisymmetric eigenvalue of $H$, i.e. $E_{0, A}$, we choose $\gamma>0$ large enough so that $E_{0, A}^{\prime}$ is by far the lowest eigenvalue of $H^{\prime}$. Thus one imposes the relations

$$
E_{0, A}^{\prime}=E_{0, A}+\gamma \lambda_{A}<E_{n, \Gamma}+\gamma \lambda_{\Gamma} \quad \forall n, \quad \forall \Gamma=S, M,
$$


which corresponds to

$$
\gamma>\frac{E_{0, A}-E_{n, \Gamma}}{\lambda_{\Gamma}-\lambda_{A}}
$$

Assuming that $E_{0, A}<0$, and using the fact that $\lambda_{\Gamma}-\lambda_{A} \geq A$, the following condition is sufficient

$$
\gamma>\frac{|\mathcal{E}|}{A}
$$

Thus, with a proper value of $\gamma$ the lowest eigenstate of the Hamiltonian matrix $H^{\prime}$ is the physical antisymmetric wave-function, and the correct value of the ground-state energy $E_{0, A}$ is obtained by subtracting $\gamma \lambda_{A}$ from $E_{0, A}^{\prime}$. Of course with a proper choice of $\gamma$ one can also calculate excited states. To this end, there is no need to calculate more than a few eigenstates of the Hamiltonian matrix, and one can use the Lanczos algorithm.

\section{DISCUSSION OF RESULTS}

Within the present formalism we have calculated ground-state energies and radii of ${ }^{4} \mathrm{He}$ and ${ }^{6} \mathrm{Li}$ with various NN potentials using the following models: Volkov [6] (central), MTI/III [8] and Minnesota (MN) [9] (central spin-isospin dependent); in addition for ${ }^{4} \mathrm{He}$ we have used AV4' [10] (central spin-isospin dependent) and the realistic AV18 [5]. In all our calculations the Coulomb force is included and the isospin mixing is neglected. Since for Volkov, MN, and MTI/III interactions there is no unique parameter setting we define these potential models in Table \.

To illustrate the typical size of the HH basis, in Table I we list its dimension for increasing $K_{\max }$. It is evident that the number of basis functions grows very rapidly. Comparing for example the dimensions with $K_{\max }=10$, one sees that for ${ }^{4} \mathrm{He}$ the number of $\mathrm{HH}$ states grows from about 1000 for a central interaction to about 5000 for a noncentral potential. For ${ }^{6} \mathrm{Li}$ one already starts with about 500000 states with a central force which is then increased to about 7 million states with a noncentral potential. Including the hyperradial part the size of the Hamiltonian matrix increases further. In fact the numbers in the table have to be multiplied by a factor 20 which is the number of Laguerre polynomials that we consider for each $K$.

To improve the convergence we have used the EIHH (Effective Interaction Hyperspherical Harmonics) formalism [11]. To distinguish between the calculations without and with effective interaction we use the acronyms NSHH and EI-NSHH, respectively. The use of 
TABLE I: Definition and parameter setting for Volkov, MN, and MTI/III NN potentials. Volkov: $V(r)=V_{1} \exp \left[-\left(r / \mu_{1}\right)^{2}\right]+V_{2} \exp \left[-\left(r / \mu_{2}\right)^{2}\right] ; \mathrm{MN}: V(r)=V_{1} \exp \left(-\mu_{1} r^{2}\right)+V_{2} \exp \left(-\mu_{2} r^{2}\right)$ and $V(r)=V_{1} \exp \left(-\mu_{1} r^{2}\right)+V_{3} \exp \left(-\mu_{3} r^{2}\right)$ for NN channels with spin $S=0$, isospin $T=1$ and $S=1, T=0$, respectively; MTI/III: $V(r)=V_{1} \exp \left(-\mu_{1} r\right) / r+V_{2} \exp \left(-\mu_{2} r\right) / r(S=0, T=1)$, $V(r)=V_{1} \exp \left(-\mu_{1} r\right) / r+V_{3} \exp \left(-\mu_{3} r\right) / r(S=1, T=0)$. The parameters $V_{i}$ are in units of $\mathrm{MeV}$ (Volkov, MN) and of $\mathrm{MeV} \cdot \mathrm{fm}(\mathrm{MTI} / \mathrm{III})$; the parameters $\mu_{i}$ are in units of $\mathrm{fm}, \mathrm{fm}^{-2}$, and $\mathrm{fm}^{-1}$ for Volkov, MN, and MTI/III potential, respectively.

\begin{tabular}{c|cc|cc|cc}
\hline \hline & \multicolumn{2}{|c|}{ Volkov } & \multicolumn{2}{c|}{$\mathrm{MN}$} & \multicolumn{2}{c}{ MTI/III } \\
\hline $\mathrm{i}$ & $V_{i}$ & $\mu_{i}$ & $V_{i}$ & $\mu_{i}$ & $V_{i}$ & $\mu_{i}$ \\
\hline 1 & 144.86 & 0.82 & 200.0 & 1.487 & 1458.047 & 3.11 \\
2 & -83.34 & 1.60 & -91.85 & 0.465 & -520.872 & 1.555 \\
3 & - & - & -178.0 & 0.639 & -635.306 & 1.555 \\
\hline \hline
\end{tabular}

effective interaction accelerates the convergence considerably, as can be seen in Table III, where we list ${ }^{4} \mathrm{He}$ ground-state energies and root mean square (RMS) radii obtained with the Volkov and the MTI/III potentials. One notes that the use of an effective interaction is not very important for the Volkov potential, whereas it has a great effect in case of the MTI/III interaction. Considering for example the low value of $K_{\max }=4$ one obtains already results close to the converged ones for all cases, except for the MTI/III without effective interaction. The difference is explained by the fact that the Volkov force has a very soft core, whereas the MTI/III potential has a rather strong short-range repulsion, which requires $\mathrm{HH}$ functions with a rather large $K_{N}$. Thus, in order to accelerate the convergence, we use the EIHH method for all the results discussed further below. In the table we list in addition a selection of results from the literature (note that for the MTI/III potential parameter settings different from ours are also in use). Comparing with our NSHH results one observes a very good agreement.

In Table IV we list results for ${ }^{4} \mathrm{He}$ obtained with $\mathrm{MN}$ and $\mathrm{AV} 4^{\prime}$ potentials. Also in this case we find a very good convergence and a good agreement with results from other authors. As already shown in Table the number of HH states increases significantly using a realistic NN force instead of a central potential model. Nonetheless, as Table $\mathrm{V}$ illustrates, we are 
TABLE II: Number of NSHH basis functions (including spin-isospin functions, but without hyperradial part) for increasing values of $K_{\max }$, for ${ }^{4} \mathrm{He}$ and ${ }^{6} \mathrm{Li}$ with central and noncentral potentials.

\begin{tabular}{c|cc|cc}
\hline \hline & \multicolumn{2}{|c|}{${ }^{4} \mathrm{He}$} & \multicolumn{2}{c}{${ }^{6} \mathrm{Li}$} \\
\hline$K_{\max }$ & central noncentral & central & noncentral \\
\hline 2 & 24 & 54 & 675 & 2750 \\
4 & 84 & 264 & 5400 & 40025 \\
6 & 224 & 852 & 30600 & 315675 \\
8 & 504 & 2172 & 137025 & 1728950 \\
10 & 1008 & 4746 & 514215 & 7392960 \\
12 & 1848 & 9269 & 1678950 & 26377350 \\
14 & 3168 & 16776 & - & - \\
16 & 5148 & 28404 & - & - \\
18 & 8008 & 45694 & - & - \\
20 & 12012 & 79488 & - & - \\
22 & 17472 & 104988 & - & - \\
24 & 24752 & 151788 & - & - \\
\hline \hline
\end{tabular}

also able to reach convergent results for ${ }^{4} \mathrm{He}$ with the modern realistic AV18 potential. In the table we also make a detailed comparison with an EIHH calculation with symmetrized $\mathrm{HH}$ states. It is evident that both calculations lead essentially to the same results. Further results from other groups displayed in Table $\mathrm{V}$ also confirm the high precision of present-day few-nucleon calculations.

Now we turn to the ${ }^{6} \mathrm{Li}$ nucleus. In Table VI we present results for central potential models only. The reason is the non-parallel character of our present code, which prevents us from using a very large basis (in future we plan to work with a parallel code which should allow to obtain converged results for $A=6-8$ with more realistic nuclear force models). The Table shows that, similarly as for ${ }^{4} \mathrm{He}$ case, the results for the Volkov potential converge already for rather low $K_{\max }$ values. On the contrary for the MN interaction and even more for the MTI/III potential it would be desirable to make calculations for even higher $K_{\max }$ values in order to obtain a more converged result for the radius. However, our ${ }^{6}$ Li results are 
TABLE III: ${ }^{4} \mathrm{He}$ ground-state energy $E_{0}$ and radius $r_{\mathrm{RMS}}$ in units of $\mathrm{MeV}$ and fm, respectively, with Volkov and MTI/III potentials with (EI-NSHH) and without (NSHH) effective interaction.

\begin{tabular}{|c|c|c|c|c|c|c|c|c|}
\hline & \multicolumn{4}{|c|}{ Volkov } & \multicolumn{4}{|c|}{ MTI/III } \\
\hline & \multicolumn{2}{|c|}{ NSHH } & \multicolumn{2}{|c|}{ EI-NSHH } & \multicolumn{2}{|c|}{$\mathrm{NSHH}$} & \multicolumn{2}{|c|}{ EI-NSHH } \\
\hline$K_{\max }$ & $E_{0}$ & $r_{\mathrm{RMS}}$ & $E_{0}$ & $r_{\mathrm{RMS}}$ & $E_{0}$ & $r_{\mathrm{RMS}}$ & $E_{0}$ & $r_{\mathrm{RMS}}$ \\
\hline 2 & -28.579 & 1.4877 & - & - & - & - & - & - \\
\hline 4 & -29.281 & 1.4920 & -30.414 & 1.4907 & -9.679 & 1.8667 & -30.782 & 1.4236 \\
\hline 6 & -29.811 & 1.4867 & -30.417 & 1.4905 & -13.744 & 1.6401 & -30.599 & 1.4263 \\
\hline 8 & -30.160 & 1.4847 & -30.449 & 1.4901 & -19.923 & 1.4612 & -30.922 & 1.4193 \\
\hline 10 & -30.276 & 1.4854 & -30.407 & 1.4899 & -22.807 & 1.4196 & -30.584 & 1.4235 \\
\hline 12 & -30.363 & 1.4871 & -30.422 & 1.4902 & -25.808 & 1.3984 & -30.763 & 1.4221 \\
\hline 14 & -30.390 & 1.4881 & -30.416 & 1.4900 & -27.253 & 1.3938 & -30.679 & 1.4223 \\
\hline 16 & -30.405 & 1.4889 & -30.417 & 1.4900 & -28.385 & 1.3946 & -30.700 & 1.4221 \\
\hline 18 & - & - & - & - & -29.061 & 1.3978 & -30.687 & 1.4221 \\
\hline 20 & - & - & - & - & -29.558 & 1.4015 & -30.696 & 1.4220 \\
\hline 22 & - & - & - & - & -29.856 & 1.4048 & -30.687 & 1.4219 \\
\hline 24 & - & - & - & - & -30.092 & 1.4081 & -30.693 & 1.4220 \\
\hline \multicolumn{2}{|c|}{ NSHH [2] } & & -30.418 & & & & & \\
\hline \multicolumn{2}{|c|}{$\mathrm{HH}[12,13]$} & & -30.420 & 1.490 & & & & \\
\hline \multicolumn{2}{|c|}{ SVM [14] } & & -30.42 & & & & & \\
\hline EIHH $[1$ & & & & & & & -30.71 & 1.4222 \\
\hline CHH 15 & & & & & & & -30.69 & 1.421 \\
\hline
\end{tabular}

of the same level of precision as those from the literature and the agreement is satisfying.

We summarize our work as follows. The Schrödinger equation is solved for an $A$-body nucleus using an expansion of the wave function in nonsymmetrized hyperspherical harmonics. Our approach is both an extension and a modification of the formalism developed by Gattobigio et al. [1, 2]. The extension consists in the inclusion of spin and isospin degrees of freedom such that a calculation with more realistic NN potential models becomes possible. 
TABLE IV: EI-NSHH results for $E_{0}$ and $r_{\text {RMS }}$ (units as in Table III) of ${ }^{4} \mathrm{He}$ with MN and AV4' potentials.

\begin{tabular}{|c|c|c|c|c|}
\hline \multirow[b]{2}{*}{$K_{\max }$} & \multicolumn{2}{|c|}{$\mathrm{MN}$} & \multicolumn{2}{|c|}{$\mathrm{AV} 4^{\prime}$} \\
\hline & $E_{0}$ & $r_{\mathrm{RMS}}$ & $E_{0}$ & $r_{\mathrm{RMS}}$ \\
\hline 2 & -29.723 & 1.4049 & -32.258 & 1.3698 \\
\hline 4 & -30.065 & 1.4139 & -32.227 & 1.3840 \\
\hline 6 & -29.950 & 1.4112 & -31.781 & 1.3957 \\
\hline 8 & -29.981 & 1.4108 & -32.579 & 1.3798 \\
\hline 10 & -29.937 & 1.4104 & -31.858 & 1.3883 \\
\hline 12 & -29.951 & 1.4107 & -32.201 & 1.3866 \\
\hline 14 & -29.945 & 1.4104 & -32.047 & 1.3865 \\
\hline 16 & -29.946 & 1.4104 & -32.068 & 1.3865 \\
\hline 18 & -29.945 & 1.4104 & -32.051 & 1.3865 \\
\hline 20 & -29.945 & 1.4104 & -32.060 & 1.3865 \\
\hline 22 & -29.945 & 1.4104 & -32.049 & 1.3862 \\
\hline 24 & -29.945 & 1.4103 & -32.054 & 1.3863 \\
\hline EIHH [11] & -29.96 & 1.4106 & & \\
\hline $\mathrm{HH}[12,13]$ & -29.947 & 1.4105 & & \\
\hline SVM [14] & -29.937 & & & \\
\hline GFMC [10] & & & $-32.11(2)$ & \\
\hline
\end{tabular}

Different from Refs. [1, 2] the cumbersome application of projection operators in order to determine the permutation symmetry of the various eigenfunctions of the Hamiltonian $H$ is avoided. Instead, a potential term proportional to the Casimir operator $\hat{C}(A)$ is added, such that, after diagonalizing the new Hamiltonian, the state with the lowest energy is manifestly antisymmetric. Since one knows the eigenvalue of the additional potential term, and since $H$ and $\hat{C}$ commute the ground-state energy and wave function of the original $A$-body problem can be obtained in a straightforward manner. In this work the approach has been used to calculate binding energy and radii of four- and six-body nuclei $\left({ }^{4} \mathrm{He},{ }^{6} \mathrm{Li}\right)$ with various $\mathrm{NN}$ 
TABLE V: EI-NSHH results for $E_{0}$ and $r_{\text {RMS }}$ (units as in Table III) of ${ }^{4}$ He with AV18 potential.

\begin{tabular}{c|cc|cc}
\hline \hline & \multicolumn{2}{|c|}{ present work } & \multicolumn{2}{c}{ Reference [16] } \\
\hline$K_{\max }$ & $E_{0}$ & $r_{\mathrm{RMS}}$ & $E_{0}$ & $r_{\mathrm{RMS}}$ \\
\hline 2 & -24.640 & 1.5063 & - & - \\
4 & -26.124 & 1.5111 & - & - \\
6 & -25.311 & 1.5061 & -25.312 & 1.506 \\
8 & -24.999 & 1.5089 & -25.000 & 1.509 \\
10 & -24.442 & 1.5197 & -24.443 & 1.520 \\
12 & -24.491 & 1.5176 & -24.492 & 1.518 \\
14 & -24.348 & 1.5184 & -24.350 & 1.518 \\
16 & -24.313 & 1.5181 & -24.315 & 1.518 \\
18 & -24.271 & 1.5177 & -24.273 & 1.518 \\
20 & -24.266 & 1.5176 & -24.268 & 1.518 \\
22 & -24.246 & 1.5170 & - & - \\
\hline HH [12, 13] & -24.22 & 1.512 & & \\
FY [17] & -24.23 & & & \\
FY [18] & -24.22 & 1.516 & & \\
\hline \hline
\end{tabular}

potential models and it has been shown that the results agree with those from the literature.

\section{Acknowledgements}

The work of N.B. was supported by the Israel Science Foundation (Grant number 954/09). W.L. and G.O. acknowledge support from the MIUR grant PRIN-2009TWL3MX.

[1] M. Gattobigio, A. Kievsky, M. Viviani, and P. Barletta, Phys. Rev. A 79, 032513 (2009).

[2] M. Gattobigio, A. Kievsky, and M. Viviani, Phys. Rev. C 83, 024001 (2011). 
TABLE VI: EI-NSHH results for $E_{0}$ and $r_{\text {RMS }}$ (units as in Table III) of ${ }^{6} \mathrm{Li}$ with various potentials.

\begin{tabular}{|c|c|c|c|c|c|c|}
\hline & \multicolumn{2}{|c|}{ Volkov } & \multicolumn{2}{|c|}{ MTI/III } & \multicolumn{2}{|c|}{$\mathrm{MN}$} \\
\hline$K_{\max }$ & $E_{0}$ & $r_{\mathrm{RMS}}$ & $E_{0}$ & $r_{\mathrm{RMS}}$ & $E_{0}$ & $r_{\mathrm{RMS}}$ \\
\hline 2 & -75.468 & 1.6823 & -46.991 & 2.1609 & -47.402 & 2.1297 \\
\hline 4 & -66.525 & 1.5922 & -36.689 & 2.0793 & -35.712 & 2.0813 \\
\hline 6 & -66.725 & 1.6005 & -36.450 & 2.1202 & -35.277 & 2.1298 \\
\hline 8 & -66.577 & 1.5993 & -36.380 & 2.1380 & -35.067 & 2.1504 \\
\hline 10 & - & - & -36.324 & 2.1562 & - & - \\
\hline 12 & - & - & -36.269 & 2.1796 & - & - \\
\hline NSHH [2] & -66.491 & & & & & \\
\hline EIHH [11] & & & -36.6 & 2.15 & -35.2 & 2.1 \\
\hline $\mathrm{HH}$ [20] & -66.57 & & -35.91 & & & \\
\hline
\end{tabular}

[3] W. Leidemann and G. Orlandini, Progr. Part. Nucl. Phys. 68, 158 (2013).

[4] N. Barnea and A. Novoselsky, Phys. Rev. A 57, 48 (1998); Ann. Phys. (N.Y.) 256, 192 (1997).

[5] R.B. Wiringa, V.G.J. Stoks, and R. Schiavilla, Phys. Rev. C 51, 38 (1995).

[6] A.B. Volkov, Nuclear Physics, 74, 33 (1965).

[7] D.H. Gloeckner and R.D. Lawson, Phys. Lett. B 53, 313 (1974).

[8] R.A. Malfliet and A. Tjon, Nucl. Phys. A 127, 161 (1969).

[9] D.R. Thomson, M. LeMere, and Y.C. Yang, Nucl. Phys. A 286, 53 (1977).

[10] R.B. Wiringa and S.C. Pieper, Phys. Rev. Lett. 89, 182501 (2002).

[11] N. Barnea, W. Leidemann, and G. Orlandini, Phys. Rev. C 61, 54001 (2000); Nucl. Phys. A 693, 565 (2001).

[12] M. Viviani, A. Kievsky and S. Rosati, Phys. Rev. C 71, 24006 (2005).

[13] A. Kievsky, S. Rosati, M. Viviani, L.E. Marcucci and L. Girlanda, Journal of Physics G: Nuclear and Particle Physics, 35, 63101 (2008).

[14] K. Varga and Y. Suzuki, Phys. Rev. C, 52, 2885 (1995).

[15] N. Barnea, V.D. Efros, W. Leidemann, and G. Orlandini, Phys. Rev. C 63, 057002 (2001).

[16] D. Gazit, S. Bacca, N. Barnea, W. Leidemann and G. Orlandini, Phys. Rev. Lett. 96, 112301 
(2006).

[17] A. Nogga, H. Kamada, W. Glöckle, and B.R. Barrett, Phys. Rev. C 65, 054003 (2002).

[18] R. Lazauskas and J. Carbonell, Phys. Rev. C 70, 044002 (2004).

[19] A. Deltuva and A.C. Fonseca, Phys. Rev. C 75, 014005 (2007).

[20] N. Barnea, W. Leidemann, and G. Orlandini, Nucl. Phys. A 650, 427 (1999). 